\title{
O USO DAS TERAPIAS ALTERNATIVAS POR ENFERMEIROS DOCENTES
}

\author{
THE USE OF ALTERNATINE THERAPIES BY NURSING TEACHERS \\ EL USO DE LAS TERAPIAS ALTERNATINAS POR ENFERMEROS DOCENTES
}

\section{Maria Julia Paes da Silva ${ }^{1}$ Maria Antonieta Benko ${ }^{2}$}

\begin{abstract}
RESUMO: Os estudos e discussões sobre as Práticas Alternativas de Saúde (PAS) estão mais freqüentes no âmbito da Enfermagem, propiciando discussões sobre o processo saúde-doença num paradigma diferente do modelo biomédico vigente. O objetivo deste trabalho foi verificar quais as práticas alternativas utilizadas por docentes de Enfermagem em si mesmos e/ou nas respectivas famílias e se as discutem com seus alunos. A população do estudo foi 59 docentes de graduação em Enfermagem da cidade de São Paulo, que responderam a um questionário. Os respondentes foram escolhidos aleatoriamente e os dados coletados em outubro de 1996. Verificou-se que $39(66,1 \%)$ deles usaram as PAS sendo as mais comuns a homeopatia (24 vezes), os florais (21) e a massagem oriental (13). Mesmo os que não fizeram uso das PAS, são favoráveis ao seu uso em situações específicas; 56 $(94.9 \%)$ docentes são favoráveis ao seu ensino: apenas 31 (52.5\%) falam sobre isso com seus alunos.
\end{abstract}

PALAVRAS-CHAVE: Práticas alternativas - Uso por enfermeiros - Holismo.

ABSTRACT: The necessity of the health professionals to understand the health illness process in a different paradigm of the valid bio-medical model is more and more present. The objective of this work was verify what are the alternatives practices utilised for Nursing teachers for themselves and/or in their respective family relatives and if these teachers have been utilised the practices and if they talk about this subject with their students. The population of this study were 59 Nursing graduate teachers from São Paulo City placed in four different teaching institutions that answered a questionnaire. The people that answered the questionnaire were chosen at random and the data were collected in October, 1996́. Among the results, we verified that $39(66.1 \%)$ of them referred that they used the APH, in fact , the more usual alternative practice is the homeopathy (24 times), floral (21) and the eastern massage (13). The people did not use the APH are for its use in specific situations: $56(94.9 \%)$ teachers were for its teaching, but only $31(52.5 \%)$ talk about $\mathrm{APH}$ to their students.

KEYWORDS: Alternative practices - Use by nurses - Holism.

\footnotetext{
${ }^{1}$ Enfermeira. Professora e Doutora do Departamento de Enfermagem Médico-Cirúrgica da Escola de Enfermagem da Universidade de Săo Paulo.

${ }^{2}$ Enfermeira do Departamento de Enfermagem Médico-Cirúrgica da Escola de Enfermagem da Universidade de São Paulo
} 
RESUMEN: Los estudios y discusiones sobre las Prácticas Alternativas de Salud (PAS) están mas frecuentes en el ámbito de la enfermería, ocasionando discusiones sobre el proceso salud-enfermedad en una derivación diferente del modelo biomédico vigente. El objetivo de esse trabajo fué verificar cuales son las prácticas alternativas utilizadas por docentes de Enfermería en sí mismos y/o en las respectivas familias y si las discuten con sus alumnos. La población del estudio fué de 59 docentes de graduación en enfermería de la ciudad de São Paulo, que contestaron a un cuestionario. Los que contestaron fueron escogidos aleatoriamente y los datos colectados en octubre de 1996 . Se constató que $39(66.1 \%)$ de ellos usaron las PAS, siendo las mas comunes la homeopatia (24 vezes), los florales (21) y el masage oriental (13). Mismo los que no hicieron uso de las PAS, son favorables a su uso en situaciones específicas; 56 (94.9\%) docentes son favorables a su enseñanza; apenas 31 (52.5\%) hablan sobre eso com sus alumnos.

PALABRAS CLAVE: Prácticas alternativas - Uso por enfermeros - Holismo.

\section{INTRODUÇÃO}

A necessidade de os profissionais da saúde entenderem o processo saúdedoença num paradigma diferente do modelo biomédico vigente é cada vez mais presente. Nesta mudança, Capra (1982) cita alguns aspectos dessa nova visão, entre eles a necessidade de adotarmos uma atitude mais equilibrada em relação ao conhecimento racional e intuitivo; o reconhecimento de que o meio ambiente criado por nosso sistema social e econômico tornou-se uma ameaça à nossa saúde; a existência e importância da natureza subjetiva no conceito de saúde, também fortemente influenciada pelo contexto cultural em que é definida. Afirma que já está ocorrendo uma concepção cada vez mais holística e ecológica do mundo, em todos os meios, e que o universo não é mais visto como uma máquina, mas como um sistema vivo. Essa nova concepção enfatiza a interrelação e interdependência essenciais de todos os fenômenos.

Gerber (1988) refere que a medicina atual, baseada no modelo de realidade newtoniana, considera, entre outros aspectos, que a fisiologia e o comportamento psicológico do ser humano dependem da máquina estrutural do cérebro, o que nos dá, a compreensão de que energia é manifestação da matéria, e que o corpo é uma máquina complexa. Propõe um novo ponto de vista no qual a matéria é vista como uma manifestação da energia, baseado no paradigma einsteiniano e da física quântica, que reconhece toda a matéria como energia; dá, a essa nova visão de medicina, o nome de medicina vibracional. Essa visão considera o ser humano como um organismo multidimensional constituído de sistemas físicos/celulares em interação dinâmica com complexos campos energéticos reguladores. Refere, ainda, que, para entendermos melhor este raciocínio, devemos lembrar das imagens holográficas, ou seja, quando a luz laser incide sobre um objeto e produz uma fotografia tridimensional, demonstrando um princípio da natureza, o de que cada parte contém o todo. 
Brennan (1993) enfoca sete princípios a respeito da natureza da realidade que a visão holográfica nos fornece:

$1^{\circ}$ A consciência é a realidade básica - portanto qualquer ciência ou sistema de saúde baseado no mundo físico se fundamenta em causas secundárias e não primárias;

$2^{\circ}$ Todas as coisas estão ligadas a todas as outras - nenhum acontecimento, seja ele político, psicológico, atômico, deve ser considerado uma ocorrência isolada. Tudo o que fazemos, dizemos, pensamos e acreditamos a respeito de saúde e da doença afeta todas as pessoas imediatamente. Curando a nós mesmos, curamos o outro;

$3^{\circ}$ Cada parte contém o todo - tudo o que existe está dentro de nós. Ao explorar o nosso interior, também exploramos o universo;

$4^{\circ}$ O tempo é holográfico - estamos todo o tempo em toda a parte, sempre. Cada um de nós esta ligado ao "EU" que era muito saudável antes da doença, portanto, "assumindo" nossa completa totalidade, podemos curar a nós mesmos;

$5^{\circ} \mathrm{A}$ individuação e a energia são fundamentais para o universo - cada um de nós é diferente de qualquer outro ser. Nossa experiência é única e não pode ser determinada por probabilidade estatística, sem considerarmos o fator criativo inerente ao aspecto individual;

$6^{\circ} \mathrm{O}$ todo é maior que a soma das partes - cada aspecto existe dentro de outro sistema maior que ele, que tem conhecimento de todos os outros sistemas, ligando e integrando ao todo suas menores partes;

$7^{\circ} \mathrm{A}$ consciência cria a realidade e sua própria experiência de realidade isso significa que experimentamos as coisas de acordo com nossa expectativa, que por sua vez é baseada nas nossas crenças e no que herdamos geneticamente e culturalmente.

Nesses princípios, encontramos referências que auxiliam na compreensão da ação das terapias alternativas, que atuam com o aspecto sutil e holistico do ser humano.

Quanto à diferença de paradigmas em relação aos sistemas médicos, deve ser salientada a constatação decorrente dessas diferenças, de objetos e objetivos distintos das terapias "energéticas" face à ocidental/oficial. Enquanto a medicina ocidental/oficial tem por objeto a doença (por meio da identificação das patologias) e por objetivo o combate e a eliminação das doenças, as terapias homeopática, chinesa, ayurvédica, entre outras, têm como objeto o sujeito desequilibrado (o doente) e por objetivo o restabelecimento de sua saúde, ou mesmo sua ampliação. Em outras palavras, no primeiro caso, a categoria central é a doença, a patologia; no segundo caso, a categoria central é a saúde, o equilibrio, a harmonia. 
Outro aspecto, a nosso ver importante nessa concepção, é a visão de que as pessoas têm participação ativa tanto no seu processo de doença como no de cura, transmitindo a idéia de responsabilidade do indivíduo neste processo saúde-doença.

Ao separar o corpo de nossas emoções, como se elas não nos influenciassem, buscamos fora de nós as explicações de nossos sintomas, bem como a solução. O modelo de saúde atual responde a esta postura com diagnósticos e condutas, dando a entender que, se a pessoa não as cumprir, a piora é certa e que é necessariamente a única solução possível. Ao assumirmos a responsabilidade pela nossa cura, a própria reflexão do motivo do sintoma se transforma num processo de autoconhecimento. Nessa compreensão, buscamos alguém que estabeleça um relacionamento amigável e produtivo no sentido de potencializar a capacidade de cura inerente a todo ser humano, acrescido à do terapeuta.

A enfermeira não pode ficar alheia a esse movimento por vários motivos. Devido a natureza do seu trabalho, em contato direto com a população, quer em hospitais ou centros de saúde, é possível esclarecer a população acerca dessas práticas benéficas ou nocivas à saúde. Em um país como o nosso, onde a propaganda e a venda de remédios se faz sem muito controle por parte das autoridades sanitárias, e onde os veículos de comunicação estão, todos os dias, a divulgar métodos de prevenção e de cura de doenças, muitas vezes prejudiciais, esse papel educativo é muito importante (Nogueira, 1983).

Outro motivo, que nos leva a refletir sobre a importância de a enfermeira conhecer essas práticas, é que elas são eficazes quando usadas corretamente, ocasionam menos efeito colateral e são menos invasivas. Soma-se a isso o fato do alto custo da assistência médica privada, o alto custo dos medicamentos e a precariedade da assistência prestada pelos serviços públicos, em geral.

As práticas ou terapias, chamadas alternativas nesse trabalho, são as práticas de saúde que, em determinado momento contextual (de tempo e de espaço), assumem posição diferente da chamada medicina oficial, em nosso meio. São alternativas às terapias ortodoxas ocidentais, profissionalizantes. Exemplificando, sob esse ponto de vista, a acupuntura não seria chamada de alternativa em seu país de origem, a China, por ser ensinada em cursos oficiais; mas aqui, no Brasil, ela ainda o é (Souza; Silva, 1991).

A Organização Mundial de Saúde - OMS, em um trabalho de resgate dessas terapias, agrupa-as sob a denominação de medicina tradicional, abrangendo desde o uso de plantas medicinais e benzimentos até a ioga e a astrologia (OMS, 1978).

Barbosa (1994), em seu estudo sobre a utilização de Terapias Alternativas pelos enfermeiros brasileiros, utilizou o agrupamento das Terapias Alternativas proposto por Hill (s.d.), em: 
- TERAPIAS FISICAS - acupuntura, moxabustão, shiatsu (e outras massagens), do-In, argiloterapia, cristais.

- HIDROTERAPIA - hidroterapia(não especificada), banhos, vaporização e sauna.

- FITOTERAPIA - fitoterapia (não especificada), ervas medicinais, florais.

- NUTRIÇÃO - nutrição alternativa (não especificada), terapêutica nutricional ortomolecular

- ONDAS, RADIAÇŐES E VIBRAÇÕES - radiestesia/radiônica.

- TERAPIAS MENTAIS E ESPIRITUAIS - meditação, relaxamento psicomuscular, cromoterapia, toque terapêutico, visualização positiva, reich.

- TERAPIAS DE EXERCÍCIOS INDIVIDUAIS - biodança, vitalização.

Desde 1975, temos informes de que a cura/cuidado através de processos energéticos é estudada na Enfermagem da América do Norte, por Krieger (1979), tendo sido introduzido o ensino do Toque Terapêutico (imposição de mãos) em diferentes universidades. No Brasil, Nogueira (1983) é um expoente na área de Práticas Alternativas na Enfermagem, defendendo sua tese de livredocência sobre a fitoterapia. Barbosa (1993) verificou que 47 enfermeiros já estão atuando nessa área, utilizando uma ou mais terapias alternativas no exercício da profissão.

Mas esse momento de "redescoberta" e aceitação das terapias alternativas não ocorre só na Enfermagem, ou só na saúde. Independente de politicas sócioeconômicas ou de saúde, de seitas religiosas ou agrupamentos afins, cada vez mais encontramos propostas e preocupação com a reintegração do homem com a natureza e consigo próprio como opção de vida, em todos os hemisférios.

A OMS (1978) pontua que os sistemas tradicionais ("alternativos" para nós, profissionais de saúde brasileiros) continuam sendo a principal fonte de assistência sanitária para dois terços da população mundial e que, em determinados paises em desenvolvimento, como a China e a Índia, têm sido feito avanços impressionantes mediante a integração dos sistemas oficiais e alternativos.

Luz (1993) também afirma que podemos verificar uma tendência na prática de atendimento ocidental de incluir no seu arsenal terapias alternativas como a acupuntura, a moxabustão, a prática de exercícios de meditação ou artes marciais, por exemplo. Porém, lembra que essa inclusão é, na maioria das vezes, mera apropriação mecânica de aspectos terapêuticos que são parte de um sistema coerente e integrado. Verifica-se, nesses casos, uma descontextualização das práticas orientais, com um conseqüente desprezo pelo significado filosófico e terapêutico dessas práticas. 
Entretanto, o esforço para o reconhecimento de eficácia nesses aspectos, e a tentativa de comprová-los experimentalmente no sentido de legitimá-los, não deve ser menosprezado. Vemos o reconhecimento da terapêutica homeopática por muitos médicos de especialidades diversas, tendo inclusive uma significação político-institucional o fato da homeopatia já ser uma "especialidade médica" há mais de uma década no Brasil.

Preocupadas em desenvolver uma visão "universitária" de seus alunos sobre o processo saúde-doença, docentes da Escola de Enfermagem da Universidade São Paulo desenvolveram, de 1989 a 1993, cursos extracurriculares sobre "PRÁTICAS ALTERNATIVAS" para os alunos de graduação de Enfermagem, com o objetivo de oferecer uma visão destas práticas mais comuns no cuidado com a saúde e divulgar a experiência de alguns especialistas de reconhecido saber nesse campo. A partir de 1994, este curso passou a ser oferecido como disciplina optativa no Curso de Graduação, oferecendo 30 vagas, atualmente. A procura por esta disciplina é superior ao número de vagas oferecidas, mostrando que existe interesse dos alunos pelo tema.

O papel das universidades é o de ser um facilitador nas discussões do que tem sido realizado pela sociedade, além de desenvolver pesquisas e profissionais críticos e conscientes do seu trabalho e do seu papel profissional e social. Com essa perspectiva, nos questionamos sobre como estaria o uso das Terapias Alternativas por enfermeiros docentes, uma vez que, empíricamente, sabíamos de vários docentes que recorrem às práticas alternativas no seu autocuidado.

Fortalecendo essa compreensão, o Conselho Federal de Enfermagem, no Parecer CTA $n^{\circ}$ 004/95 sobre Atividades em Terapias Alternativas pelos profissionais de Enfermagem, reconhece que:

- a profissão de Enfermagem fundamenta-se na visão integral do ser humano, apesar de sua prática estar direcionada para o modelo biomédico;

- muitos profissionais de Enfermagem vêm demonstrando interesse em aprofundar seus conhecimentos no que diz respeito à mudança de paradigma que melhor se adeqúe a esta visão de totalidade do ser humano;

- há profissionais de enfermagem utilizando as práticas naturais no cuidado à clientela e no ensino da profissão;

- estudos acerca das práticas naturais utilizadas pela Enfermagem comprovam o interesse dos profissionais de Enfermagem em abordar estas práticas;

- vários aspectos do Código de Ética dos Profissionais de Enfermagem justificam a prática das Terapias Naturais de saúde por profissionais de Enfermagem, desde que possuam a comprovação de formação básica em tais terapias, proporcionando o desempenho seguro para si e para o cliente. 
Além deste Parecer Normativo, a Resolução COFEn - 197, de19/3/1997, "Estabelece e reconhece as Terapias Alternativas como especialidade e/ou qualificação do profissional de Enfermagem". Determina, para essa titulação, que o profissional tenha concluido e sido aprovado em curso reconhecido por instituição de ensino ou entidade congênere, com uma carga horária mínima de 360 horas.

Visto o respaldo oferecido pelo COFEn e nossa postura filosófica que aceita esse paradigma como um avanço na compreensão da totalidade do ser humano, temos como objetivos neste trabalho:

- verificar quais as práticas alternativas já utilizadas por docentes de Enfermagem em si mesmos e/ou nas respectivas famílias; e,

- se esses docentes, por utilizarem essas práticas, as discutem com seus alunos.

\section{MÉTODO}

Local de estudo - Quatro instituições de ensino de graduação em Enfermagem, duas públicas e duas particulares, da cidade de São Paulo.

População - Foram distribuídos, aleatoriamente, questionários/ instituição para os Enfermeiros docentes presentes, que aceitaram participar dessa pesquisa. A maioria preencheu-o no momento em que a solicitação foi feita, e tivemos como total a devolução de 59 questionários.

Procedimento - Foi explicado o motivo do levantamento para os docentes e, aos que aceitaram participar, foi entregue um questionário com seis questões fechadas e semi-abertas (Anexo I) perguntando sobre o uso das práticas alternativas, sua opinião sobre essas práticas no uso pessoal e no ensino e, para os que já usaram qualquer das práticas, em que situação a(s) usou, se recomenda seu uso e se discute sobre isso com os alunos. Foi deixado um espaço em branco para qualquer comentário que o docente julgasse pertinente. Os dados foram coletados durante o mês de outubro de 1996.

\section{APRESENTAÇÃO DOS RESULTADOS}

Dos $59(100 \%)$ docentes que responderam ao questionário, $39(66,10 \%)$ referiam ter feito ou fazer uso de uma ou mais das terapias alternativas (T.A.), enquanto que $20(33,90 \%)$ afirmaram não fazer uso delas. Percebemos que é uma porcentagem alta a de docentes que conhecem as práticas alternativas, pois já fizeram uso de alguma delas. Questionados sobre qual prática haviam utilizado, tivemos 82 citações, dando uma média de 1,39 prática/docente, sendo que 18 deles $(30,51 \%)$ referiram utilizar 2 práticas, $10(16,95 \%)$ uma única e 6 $(10,17 \%)$ citaram três práticas.

A prática alternativa mais citada pelos docentes foi a homeopatia (24 vezes), seguida de essências florais (21 vezes), massagem oriental (13), meditação (7), 
cristais (4), biodança e acupuntura (3 cada) e terapia ortomolecular, toque terapêutico, iridologia, fitoterapia, moxabustão, shantala e exercício de energização que tiveram somente uma citação. Mantendo o agrupamento proposto por HILL (s/d), temos o que pode ser observado na Tabela1.

TABELA 1 -RELAÇÃO DAS TERAPIAS ALTERNATIVAS USADAS POR 59 ENFERMEIROS DOCENTES. SÃO PAULO, 1996.

\begin{tabular}{|c|c|c|}
\hline \multirow{2}{*}{ GRUPOS DE TIPOS DE TERAPIAS } & \multicolumn{2}{|c|}{ Docentes que utilizam (ram) } \\
\hline & $\mathbf{F i}$ & F\% \\
\hline $\begin{array}{l}\text { 1. CONCEPÇĀO GLOBAL } \\
\text { 1.1. Homeopatia } \\
\text { 2. FITOTERAPIA } \\
\text { 2.1. Florais } \\
\text { 2.2. Fitoterapia } \\
\text { 3. TERAPIAS FISICAS } \\
\text { 3.1. Massagem Oriental } \\
\text { 3.2. Cristais } \\
\text { 3.3. Acupuntura } \\
\text { 3.4. Moxabustão } \\
\text { 3.5. Shantala } \\
\text { 4. TERAPIAS MENTAIS E ESPIRITUAIS } \\
\text { 4.1. Meditaçăo } \\
\text { 4.2. Toque Terapêutico } \\
\text { 4.3. Exercicio de energização } \\
\text { 5. TERAPIA DE EXERCICIOS INDIVIDUAIS } \\
\text { 5.1. Biodança } \\
\text { 6. METODO DE DIAGNOSE } \\
\text { 6.1. Iridologia } \\
\text { 7. NUTRIÇÃO } \\
\text { 7.1. Terapia Ortomolecular }\end{array}$ & $\begin{array}{c}13 \\
4 \\
3 \\
1 \\
1 \\
7 \\
1 \\
1 \\
3 \\
1 \\
1 \\
1\end{array}$ & $\begin{array}{c}29,27 \\
25,61 \\
1,22 \\
15,85 \\
4,88 \\
3,66 \\
1,22 \\
1,22 \\
8,53 \\
1,22 \\
1,22 \\
3,66 \\
1,22 \\
1,22 \\
\end{array}$ \\
\hline
\end{tabular}

Pela Tabela 1, podemos verificar que, como nos achados de Barbosa (1994), os números dos grupos das terapias físicas e o de fitoterapia continuam mais altos entre os enfermeiros. A homeopatia só pode ser utilizada com acompanhamento médico, por isso não apareceu como item na pesquisa de Barbosa (1994), que se dispôs a verificar em que práticas os enfermeiros estavam atuando; porém, neste trabalho, onde foi perguntado se os docentes fizeram uso de Terapias Alternativas como paciente, ela foi o item mais citado.

Questionados sobre "você é contrário ou a favor do uso de T.A.", 57(96, $61 \%$ ) docentes se pronunciaram a favor, 1 referiu não ter opinião formada e 1 deixou em branco a questão. Essa porcentagem parece-nos demonstrar que os docentes realmente estão abertos ao paradigma holístico, sentindo a necessidade de entenderem o processo saúde-doença num modelo diferente do biomédico vigente, respeitando a liberdade das pessoas que desejam optar por essas formas de prevenção e tratamento de doenças. 
Da mesma maneira, quando questionados a respeito do ensino das T.A., 56 $(94,91 \%)$ docentes referem não ser contrários ao seu ensino, 1 se posicionou contrário, 1 deixou em branco a questão e 1 referiu não ter opinião formada a respeito, É importante lembrar que essas T.A., de uma maneira geral, enfatizam que as pessoas têm participação ativa tanto no seu processo de doença como no de cura, transmitindo a idéia de responsabilidade individual nesse processo. Um docente que aceite esse tipo de ensino, podemos inferir, está mais atento ao paciente como um ser único, responsável, que precisa ser ouvido constantemente no seu tratamento, pois essa é uma das premissas dessas práticas.

Dos 39 docentes que fizeram ou fazem uso das T.A., tivemos 53 situações descritas de quando as utilizaram, sendo que $28(71,79 \%)$ deles o fizeram em uso próprio, $4(10,26 \%)$ no atendimento de outras pessoas, $1(2,56 \%)$ na pesquisa e $1(2,56 \%)$ no ensino de neonatologia. Os outros 5 docentes não especificaram a situação. Podemos perceber que, nessa população estudada, ainda são poucos os docentes que se aprofundaram nessas práticas, a ponto de utilizarem-nas também com outras pessoas, no ensino ou na pesquisa. Essa população ainda está vivenciando as T.A. em si mesma.

Das 53 situações descritas pelos docentes, 29 (54,72\%) estão relacionadas ao aspecto mais físico das doenças (processos inflamatórios, alergias, doenças crônicas, dores, menopausa, entre outros), 17 (32,10\%) estão relacionadas mais ao mental ("stress", depressão, ansiedade e desânimo), $4(7,55 \%)$ mais ao emocional ("momentos difíceis", morte na família) e $3(5,66 \%)$ a situações mais abrangentes, rotineiras, que envolvem práticas de prevenção e harmonização.

As três práticas mais citadas pelos docentes no atendimento de qualquer um desses aspectos (físico, mental, emocional) foram a homeopatia, os florais e a massagem oriental.

O total de docentes que recomendam o uso de Práticas Alternativas, independente de já as ter utilizado ou não, pode ser verificado na Tabela 2.

TABELA 2 - DOCENTES QUE RECOMENDAM O USO DE PAS, COM E SEM EXPERIÊNCIA PESSOAL. SÃO PAULO, 1996.

\begin{tabular}{c|c|c|c}
\hline Resposta & Utilizam PAS & Năo utilizam PAS & TOTAL \\
\hline Sim & 37 & 7 & 44 \\
\hline Năo & 2 & 4 & 6 \\
\hline TOTAL & 39 & 11 & 50 \\
\hline
\end{tabular}

* 9 não responderam

Pela Tabela 2, podemos observar que dos 39 docentes que se utilizaram de T.A., 37 deles as recomendam, enquanto que 2 afirmaram não recomendar por não conhecer o suficiente sobre elas e por não ser especialista no assunto. Dos 20 docentes que afirmaram nunca ter utilizado T.A., 11 se posicionaram quanto a essa questão, sendo que 7 as recomendam, mesmo sem as ter usado, enquanto que 4 não. Os motivos registrados pelos que não utilizaram as T.A. para recomendá-las, são em casos especiais (alergias, problemas emocionais), situações estressantes (por serem relaxantes), doença crônica, entre outros. 
Com relação ao motivo da recomendação do uso, pelos docentes que fazem ou fizeram uso das PAS, encontramos 22 docentes referindo ser pela eficácia, 10 relacionaram o argumento com o tratamento convencional (não ter contraindicação, complemento do tratamento alopático, mobilizarem outros centros "energéticos" diferentes da medicina tradicional, por ser natural, agredir menos o organismo, evitar o uso de medicamento alopático) e 5 por compreenderem esta forma de intervenção como um processo participativo (permitindo voltar-se mais para o próprio interior, permitindo maior equilíbrio físico/mental). A crença na resolubilidade das T.A., que foi uma das categorias mais freqüentes no estudo dos enfermeiros brasileiros desenvolvido por Barbosa (1994), foi também o motivo mais citado pelos docentes. O desencanto com a alopatia e o desejo de trabalhar em outro paradigma também surge quando os docentes comparam o tratamento convencional com as T.A.

Questionados sobre "quando" recomendam as PAS, surgiram 24 citações em situações abrangentes (quando necessário, "em situações que foram úteis para mim", em situações sem gravidade);6 na vigência de problemas físicos (dor, edema, alergia, nervosismo, tratamento profilático ou mesmo com a doença instalada); 4 relacionadas ao aspecto mental/emocional (para autoconhecimento, auto-ajuda, stress); 2 referiram usar quando a pessoa acredita na terapia proposta e 1 especificou a necessidade do preparo do profissional para o seu uso. Chama a atenção o número de docentes que recomendam o uso das T.A. em situações abrangentes, onde o objeto de tratamento é 0 indivíduo em desequilíbrio, e não especificamente a doença. $O$ total de enfermeiros docentes que fala sobre as PAS com os alunos pode ser visto na Tabela 3

TABELA 3 - DOCENTES QUE FALAM SOBRE AS PAS COM OS SEUS ALUNOS. SÃO PAULO, 1996.

\begin{tabular}{c|c|c|c}
\hline Resposta & Utilizam PAS & Nảo utilizam PAS & TOTAL \\
\hline Sim & 23 & 3 & 26 \\
\hline Nåo & 16 & 9 & 25 \\
\hline TOTAL & 39 & 12 & 51 \\
\hline
\end{tabular}

* 8 não responderam

Observamos, pela Tabela 3, que 23 dos docentes que se utilizaram das PAS referiram falar sobre isso com seus alunos e, mesmo assim, alguns desses afirmaram que o fazem "raramente"; 16 não o fazem; dos 20 docentes que não utilizaram as PAS, 12 responderam a questão, sendo que 3 deles as discutem com seus alunos em situação de curativo, cuidado com o recém-nascido e na assistência junto à coletividade.

As situações citadas pelos 23 docentes que falam sobre esse assunto com seus alunos foram 12 em situação de ensino e 11 em situações não diretamente ligadas ao ensino formal ("quando surge oportunidade"). Podemos perceber que é pequeno o número de docentes que discutem PAS com seus alunos, o que a nosso ver prejudica a formação do futuro profissional de Enfermagem, que deixa de receber uma visão mais abrangente das possibilidades de sua atuação. 
Foi deixado um espaço para que os docentes fizessem outro comentários sobre o assunto e tivemos 20 questionários com observações, sendo 14 de docentes que utilizam as PAS e 6 dos que não as utilizam. A maioria dos registros refere-se à importância das PAS, porém desde que haja respaldo técnico-científico correspondente. Valorizam 0 assunto e consideram-no importante na formação do futuro enfermeiro. Exemplificando:

"Acredito, apesar do pouco conhecimento na área, que algumas PAS podem melhorar tanto a assistência como o ensino na area de enfermagem, podem e devem ser realizadas com pessoas qualificadas e com respaldo científico"( não utiliza as PAS);

"Embora acredite nas PAS, ainda não havia me alertado para transmiti-la, abordá-la com os alunos. A partir de agora ficarei mais atenta com relação a essas práticas e transmissão das mesmas";

"Creio que são conteúdos importantíssimos para a prática do Enfermeiro, uma vez que abre uma nova área profissional e amplia as possibilidades de superação dos agravos à saúde".

\section{CONCLUSÕES E CONSIDERAÇÕES FINAIS}

Diante dos objetivos propostos, verificamos que, dos 59 docentes questionados, 39 deles referiram ter feito uso das PAS, sendo as mais comuns a homeopatia (24 vezes), os florais (21) e a massagem oriental (13). Mesmo os que não fizeram uso das PAS são favoráveis ao seu uso em situações especificas.

56 docentes referiram ser favoráveis ao seu ensino, mas apenas 31 (52,5\%) falam sobre isso com seus alunos, denotando uma falha na formação dos futuros profissionais, no que se refere a essa temática.

O novo paradigma (é ainda novo?) já existe e cresce nas diferentes áreas sociais. A saúde e a Enfermagem não estão alheias a esse processo, mesmo porque está claro nessa concepção, o inter-relacionamento e a interdependência entre todos os fenômenos.

Os órgãos formadores estão entendendo a necessidade dessas discussões ocorrerem no interior das academias para que não percamos a seriedade que merece o tratar dos seres humanos. Vamos oficializar e intensificar essas discussões, em nome da própria riqueza que é a vida, o ser humano, nas suas diferentes facetas. 


\section{REFERÊNCIAS BIBLIOGRÁFICAS}

1. BARBOSA, M. A.. A utilização de terapias alternativas por enfermeiros brasileiros. São Paulo,1994. 259p. Tese (Doutorado em Enfermagem) - Escola de Enfermagem, Universidade de São Paulo.

2. BRENNAN, B. A .. Luz emergente: a jornada da cura pessoal, São Paulo: Cultrix, 1993.

3. CAPRA, F.. O ponto de mutação. São Paulo: Cultrix, 1982.

4. CONSELHO FEDERAL DE ENFERMAGEM. Parecer Normativo do COFEn no 004/95. Dispõe sobre as atividades em Terapia Alternativa. Bol. Inf. COREn, v.18, n.4, p.8, 1995.

5. CONSELHO FEDERAL DE ENFERMAGEM. Resolução COFEn 197. Estabelece e reconhece as Terapias Alternativas como especialidade e/ou qualificação do profissional de Enfermagem. In: CONSELHO REGIONAL DE ENFERMAGEM. Documentos básicos de enfermagem_. São Paulo, 1997.p197.

6. GERBER, R.. Medicina vibracional: uma medicina para o futuro. São Paulo: Cultrix, 1988.

7. HILL, A .. Guia das medicinas alternativas. São Paulo, Humus, s.d.

8. LUZ, M.T.. Racionalidades médicas e terapêuticas alternativas. Rio de Janeiro, UERJ/IMS, 1993. (Série Estudos em Saúde Coletiva, 62.)

9. KRIEGER, D.. The therapeutic touch - how use your hands to help or to heal. Englewood Cliffs: Prentice Hall, 1979.

10. NOGUEIRA, M.J. de C.. Fitoterapia e enfermagem comunitária. São Paulo, 1983. 257p. Tese (Livre Docência) - Escola de Enfermagem, Universidade de São Paulo.

11. NOGUEIRA, M. J. de C.. Terapêuticas alternativas na enfermagem: por que não? Enfoque, v.11,n. 2, p.30-5, 1983.

12. ORGANIZACION MUNDIAL DE LA SALUD Promoción y desarrollo de la medicina tradicional: informe de uma reunion de la OMS.. Genebra, 1978.. (Série Informes Técnicos, 622)

13. SOUZA, D. de; SILVA, M. J. P. da. O holismo espiritualista como referencial teórico para o enfermeiro., R. EsC. Enferm. USP, v.26, n.2, p.235-42, 1992. 